\title{
Gewerkschaften als Interessenvertreter der älteren Generation?
}

Im Jahr 2009 waren rund 1,3 Mio. Rentner und Pensionäre Mitglieder in den Gewerkschaften des Deutschen Gewerkschaftsbundes (DGB). Damit gehören Gewerkschaften mit zu den größten Seniorenorganisationen in Deutschland. Das stellt sie als tarif- und betriebspolitische Interessenvertreter der Erwerbstätigen vor besondere Herausforderungen. Denn auch die Mitglieder im Ruhestand möchten interessenpolitisch vertreten werden. Der Beitrag diskutiert, wie Senioren in den Gewerkschaften derzeit integriert sind und welche Folgen sich daraus für die politische Arbeit der Gewerkschaften ergeben können.

\section{Einleitung}

Man stelle sich vor, am 1. Mai würden die Gewerkschaftssenioren ${ }^{1}$ geschlossen $\mathrm{zu}$ Hause bleiben. Ein vermutlich klägliches Bild würden die öffentlichen Kundgebungen zum Tag der Arbeit liefern. Dieses fiktive Szenario gibt einen Hinweis auf die unübersehbare Alterung der deutschen Gewerkschaften. Seit über zwei Jahrzehnten sind die Einzelgewerkschaften des DGB mit einer demografischen Veränderung ihrer Mitgliedschaft konfrontiert, die sich als „kombinierter Alterungs- und Schrumpfungsprozess" charakterisieren lässt (Streeck 2007, S. 294). Denn nach der Gruppe der Erwerbstätigen bilden die Senioren die zweitgrößte Mitgliedergruppe: Im Jahr 2009 waren $21 \%$ (absolut 1,3 Mio.) bzw. jedes fünfte Mitglied der DGBGewerkschaften Rentner oder Pensionär. ${ }^{2}$

Die beschriebene Entwicklung ist alles andere als neu. Bereits in den 1990er Jahren hielten Martin Kohli und Harald Künemund fest: „Unbeabsichtigt und weitgehend unbemerkt hat sich der Deutsche Gewerkschaftsbund mit seinen Mitgliederorganisationen zu einer der größten deutschen Seniorenorganisationen entwickelt" (Kohli/Künemund 1998, S. 731). Die Zunahme der Mitglieder außerhalb der Arbeitsgesellschaft birgt für die Erwerbstätigenorganisationen mögliche strategische Widersprüche. Denn von ihrer Entstehung und Funktion her betrachtet sind sie organisierte Zusammenschlüsse von Erwerbspersonen mit dem Zweck, die wirtschaftlichen, sozialen und politischen Interessen ihrer Mitglieder in den Arbeitsbeziehungen und im politischen System zur Geltung zu bringen, wobei die
Mitgliedschaft freiwillig ist. Sie agieren sowohl als Kampfverband in tarifpolitischen Auseinandersetzungen wie auch als politischer Verband in der sozialpolitischen Arena. Gleichzeitig begreift sich die gewerkschaftliche Solidargemeinschaft seit jeher auch als Interessenvertretung der ehemals Beschäftigten und damit als generationenübergreifende Organisation. Mit der veränderten Altersstruktur ihrer Mitgliedschaft ist jedoch zu fragen: Wandelt sich die bisherige Randfunktion, die advokatorische Mitvertretung der Interessen von Älteren, hin zu einer direkteren Interessenvertretungspolitik? Ein grundlegender Zielkonflikt hinsichtlich ihrer Kernfunktion als Arbeitnehmervertretung würde sich dann feststellen lassen, wenn die Gegenwartsinteressen der Älteren im Ruhestand mit den Zukunftsinteressen der erwerbstätigen Jungen nicht mehr im Organisationsgefüge ausbalanciert werden können.

Um potenzielle Veränderungen der gewerkschaftlichen Handlungs- und Interessenvertretungslogik zu diskutieren, resümieren wir zunächst knapp den wissenschaftlichen Diskussionsstand - fokussiert auf das Thema der gewerkschaftlichen Seniorenpolitik (Abschnitt 2) - und richten sodann den Blick auf die Mitgliederstruktur der Gewerkschaften (Abschnitt 3). Dabei wird auf die unterschiedliche Entwicklung in den drei mitgliederstärksten Gewerkschaften IG Metall, IC BCE und ver.di eingegangen. Abschnitt 4 gibt anhand ausgewählter Gewerkschaften einen Einblick, inwieweit Senioren derzeit an den inhaltlichen Schwerpunkten, Organisations- und Beteiligungsformen der Gewerkschaften partizipieren. Schließlich wird diskutiert, ob und wie das Potenzial der aktiven Älteren in den Gewerkschaften - trotz möglicher struktureller Zielkon- flikte der Gewerkschaftsarbeit - zum beiderseitigen Nutzen von erwerbstätigen und nicht erwerbstätigen Mitgliedern fruchtbar gemacht werden könnte (Abschnitt 5). Abschnitt 6 zieht ein Fazit.

1 Diese Publikation verwendet ausschließlich die männliche Sprachform. Bei allen Personen- und Funktionsbezeichnungen sind stets auch weibliche gemeint. Die Verwendung nur einer Geschlechtsform wurde für eine bessere Lesbarkeit gewählt. Unter der Bezeichnung Senioren werden Rentner, Pensionäre sowie Personen im Vorruhestand gefasst.

2 Auf den Alterungsprozess der Christlichen $\mathrm{Ge}$ werkschaften und des Deutschen Beamtenbundes (DBB) wird im Folgenden nicht eingegangen. Im Zentrum der Ausführungen steht die Entwicklung des DGB und seiner Mitgliedsgewerkschaften.

Wolfgang Schroeder, Prof. Dr., Universität
Kassel, Fachgebiet Politisches System
der Bundesrepublik Deutschland.
Arbeitsschwerpunkte: Wandel von Politik
und Ökonomie in Deutschland und Europa,
Sozialstaatlicher Umbau (Gesundheits-,
Arbeitsmarkt- und Rentenpolitik),
Vergleichende Wohlfahrtsstaatsforschung,
Parteien- und Organisationsforschung.
e-mail: wolfgang.schroeder@uni-kassel.de
Bettina Munimus, M. A., Politik-
wissenschaftlerin, Graduiertenstipendiatin
der Friedrich-Ebert-Stiftung, war
wissenschaftliche Mitarbeiterin im
Projekt „Alternde Gesellschaften und
Organisationen für Ältere. Sozialverbände
und Gewerkschaften in nationaler und
internationaler Perspektive“ an der
Universität Kassel. Arbeitsschwerpunkte:
Parteien- und Organisationsforschung,
Wandel von Politik und Ökonomie in
Deutschland und Europa insbesondere
unter der Perspektive des demografischen
Wandels, Sozialstaatsforschung.
e-mail: munimus@uni-kassel.de
Wand




\section{Gewerkschaftliche Seniorenarbeit als Forschungsfeld}

Parallel zur wachsenden Sensibilität für gesellschaftliche Alterungs- und Schrumpfungsprozesse, die sich auch in einer vom Bundestag eingerichteten Enquete-Kommission zum demografischen Wandel $^{3}$ niederschlug, begann in den 1990er Jahren die wissenschaftliche Analyse dieser Prozesse in und für die Gewerkschaften. So legten die Berliner Soziologen Jürgen Wolf, Martin Kohli und Harald Künemund (1994) eine erste umfängliche Studie zu den Voraussetzungen und Folgen einer gewerkschaftlichen Altenpolitik vor. In dieser Untersuchung befassten sie sich primär mit der Seniorenarbeit in der IG Metall. Aus der Perspektive der älteren Mitglieder untersuchten sie deren Lebenslagen sowie ihr politisches und soziales Interesse an einem Ehrenamt im gewerkschaftlichen Wirkungskreis. Ebenso wurden inhaltliche Schwerpunkte, Organisations- und Beteiligungsformen von Gewerkschaftssenioren berücksichtigt. Zum Zeitpunkt der Veröffentlichung dieser Studie war die Seniorenarbeit in zehn der damals 16 DGB-Einzelgewerkschaften satzungsrechtlich verankert. Im Ergebnis konstatieren die Autoren eine Kluft zwischen den proklamierten seniorenpolitischen Zielen und der Organisationsrealität in vielen Gewerkschaften (ebd., S. 400). Organisationsinterne Zielvorgaben stünden oft unverbunden neben oder sogar im Gegensatz zu durchaus im Interesse der Gewerkschaft liegenden externen Optionen, die funktionsentlastend und im Sinne einer Bindung der Älteren an die Organisationen wirken könnten. Gleichwohl lautete ihre Prognose: In Deutschland sei die Gründung einer separat von den DGB-Gewerkschaften agierenden Rentnergewerkschaft, wie sie in Italien besteht, nicht in Sicht (Künemund et al. 1993; Wolf et al. 1994). Trotz ihrer Entdramatisierungsthese sahen die Autoren Handlungsbedarf und plädierten für ein koordiniertes Vorgehen der Einzelgewerkschaften unter dem Dach des DGB, um den Gewerkschaftssenioren ein „quer zu den hergebrachten Branchenzuordnungen" (Wolf et al. 1994, S. 401) liegendes Organisationsangebot zu ermöglichen. Zugleich würden sie Mitglied in ihren jeweiligen Einzelgewerkschaften bleiben. Zum damaligen Zeitpunkt, so resümierten die Autoren, hinkten die deutschen Gewerkschaften einer derartigen Entwicklung-im Unterschied zu anderen Ländern in Europa ${ }^{4}$ - hinterher. Dies lässt sich auch rund 16 Jahre nach der Studie konstatieren.

Die Studie von Wolfet al. (1994) bildete den Startpunkt für die wissenschaftliche Erforschung der organisationspolitischen Interessenlagen von nichterwerbstätigen Gewerkschaftsmitgliedern, vor allem der Motiv- und Anreizstrukturen für die Beibehaltung der Mitgliedschaft im Ruhestand und den potenziellen Organisationsformen für Senioren in den unterschiedlichen Verbandsstrukturen. Einige Jahre später haben Wolf et al. (2003) die Studie "Neue Ehrenamtlichkeit in traditionellen Mitgliederverbänden“ vorgestellt. Neben dem Engagement von Jugendlichen und Erwerbslosen beschäftigten sich die Autoren ebenfalls mit den Handlungspotenzialen der gewerkschaftlichen Seniorenarbeit. Die 2010 von den Kasseler Politikwissenschaftlern Wolfgang Schroeder, Bettina Munimus und Diana Rüdt vorgelegte Studie (Schroeder et al. 2010) knüpft an den Arbeiten der Berliner an, deren Ergebnisse sie bestätigen kann, differenziert zugleich jedoch die Aktivitäten der deutschen Gewerkschaften; wobei der strukturelle Blick konsequent auf die organisationspolitischen Entwicklungen im Sinne der Interessenvertretungslogik gerichtet ist. Die Kasseler Studie weitet zudem ihre Forschungsperspektive auf die deutschen Sozialverbände aus und diskutiert, ob in Deutschland eine machtvolle eigenständige Interessenvertretung für Ältere erstarken kann, wie sie in den USA mit einem mächtigen, eigenständigen Rentnerverband oder in Italien mit selbstständigen Rentnergewerkschaften besteht. Aus dem Forschungsfundus dieser Studie ${ }^{5}$ speist sich der vorliegende Beitrag.

\section{Die Alterung der Gewerkschaften}

\subsection{EIN GENERELLES STRUKTUR- PROBLEM...}

Seit den 1970er Jahren stehen die Gewerkschaften vor massiven Herausforderungen; die damit einhergehenden strukturellen
Problemlagen wirken in vier Dimensionen: Die Mitgliederkrise, die in einem massiven Mitgliederschwund zum Ausdruck kommt, gründet sich insbesondere auf wirtschaftliche und gesellschaftliche Strukturverschiebungen. Als Folge der Mitgliederverluste sehen sich die Gewerkschaften mit einer Finanzkrise konfrontiert, die ihre Ressourcen einschränkt (vgl. Schroeder/Keudel 2008, S. 9). Die Strukturveränderungen der Betriebe, Branchen und Arbeitsmärkte forderten sie in ihrer arbeitsmarktpolitischen Kernfunktion heraus. Ebenso veränderte sich das politische System in der Weise, dass sich die Arbeitnehmervertretungen als politische Akteure angesichts des Umbaus des Sozialstaats zunehmend in einer defensiven Rolle als Verteidiger des bisherigen Systems wiederfinden. Die Aufweichung des traditionellen Sozialstaatskonsenses und der Sozialpartnerschaft führten zu einer Einbettungskrise, die darin zum Ausdruck kommt, dass die Arbeitgeberverbände sich neu ausrichteten und der Staat manche seiner etablierten Unterstützungen für die Sozialpartnerschaft zurückfuhr. Diese sich kumulierenden Veränderungen führten zu einer Durchsetzungs- und Legitimitätskrise.

Dreh- und Angelpunkt der Revitalisierungsbestrebungen, die wir gegenwärtig in den DGB-Gewerkschaften feststellen können, ist eine neue Sensibilität in der Mitgliederpolitik. Damit reagieren die Gewerkschaften nicht nur auf den absoluten Mitgliederrückgang, sondern vor allem auf die Entwicklung bei den jungen Erwerbstätigen, deren Zahl und Anteil sich seit den 1990er Jahren deutlich verringert hat, während gleichzeitig ein kontinuierlicher Alterungsprozess der verbliebenen Mitgliedschaft festzustellen

3 Zwischen 1992 und 2002 erarbeitete die EnqueteKommission „Demographischer Wandel - Herausforderungen unserer älter werdenden Gesellschaft an den Einzelnen und die Politik" Empfehlungen für politische Entscheidungen zu den gesellschaftlichen, ökonomischen und sozialen Auswirkungen, die aus dem demografischen Wandel hervorgehen

4 In Italien beispielsweise existieren eigene Rentnergewerkschaften, die ihren arbeitszentrierten Pendants an organisationaler Stärke keineswegs nachstehen.

5 Die von der Hans-Böckler-Stiftung geförderte Studie "Alternde Gesellschaften und Organisationen für Ältere. Sozialverbände und Gewerkschaften in nationaler und internationaler Perspektive" wurde von Februar 2007 bis März 2009 unter der Leitung von Prof. Dr. Wolfgang Schroeder an der Universität Kassel durchgeführt. Zu den Fragestellungen, zur Methodik und den Ergebnissen dieser empirischen Studie vgl. Schroeder et al. (2010). 
ist. Dabei steigt zwar der prozentuale Anteil der Senioren an der Gesamtmitgliedschaft, gleichzeitig nimmt aber die absolute Anzahl der Mitglieder im Ruhestand ab. Es sind also nicht die hinzugekommen Alten, sondern die fehlenden Jungen, die die Mitgliederstruktur verändern. Die Entwicklung in den DGB-Gewerkschaften wird für den Zeitraum von 1993 bis 2009 in Abbildung 1 dargestellt. Während der prozentuale Anteil der „Gewerkschaftssenioren" an der Gesamtmitgliedschaft im Jahr 1993 bei 13,3 \% lag, wuchs dieser im Zeitraum von 15 Jahren um rund neun Prozentpunkte auf $21 \%$ im Jahr 2009. Kennzeichnend für die altersstrukturelle Verschiebung ist der Anstieg dieser Mitgliedergruppe, der seinen vorläufigen Höhepunkt im Jahr 2001 fand, als der DGB insgesamt 1.667.289 Senioren organisierte. Seither hält sich der Seniorenanteil konstant. Drei Hauptfaktoren bedingen diese Dynamik: In Folge der deutschen Vereinigung stieg die Zahl der Gesamtmitglieder und auch der älteren Mitglieder zeitweilig überproportional. Zum anderen führte die in den 1990er Jahren massiv betriebene Frühverrentungspolitik zu einem starken Anstieg der Mitglieder im Ruhestand. ${ }^{6}$ Darüber hinaus haben alle Gewerkschaften anhaltende Schwierigkeiten, jüngere Arbeitnehmer zu werben: Lag in den 1980er Jahren der Anteil der 18- bis 27-Jährigen im DGB bei rund $15 \%$, so betrug er im Jahr 2009 nur noch 7,6 \%. Organisationspolitisch stehen die Arbeitnehmervertretungen daher vor der Aufgabe, eine stetig gewachsene Gruppe in die eigenen Organisationsstrukturen zu integrieren, die aus Altersgründen auBerhalb des Arbeitsmarktes steht.

\section{2 ...MIT UNTERSCHIEDLICHER AUSPRÄGUNG}

Auffallend ist, dass die Entwicklung der organisatorischen Alterungsprozesse in den einzelnen Gewerkschaften sehr unterschiedlich verläuft. So ist bei der Industriegewerkschaft Bergbau, Chemie, Energie (IG BCE) zu berücksichtigen, dass im Jahr 1997 mit der Vereinigung von IG Chemie, Papier, Keramik, der IG Bergbau sowie der Gewerkschaft Leder zur IG BCE

\section{Abb. 1: Seniorenmitgliederzahlen in den einzelnen DGB-Gewerkschaften 1993-2009*}

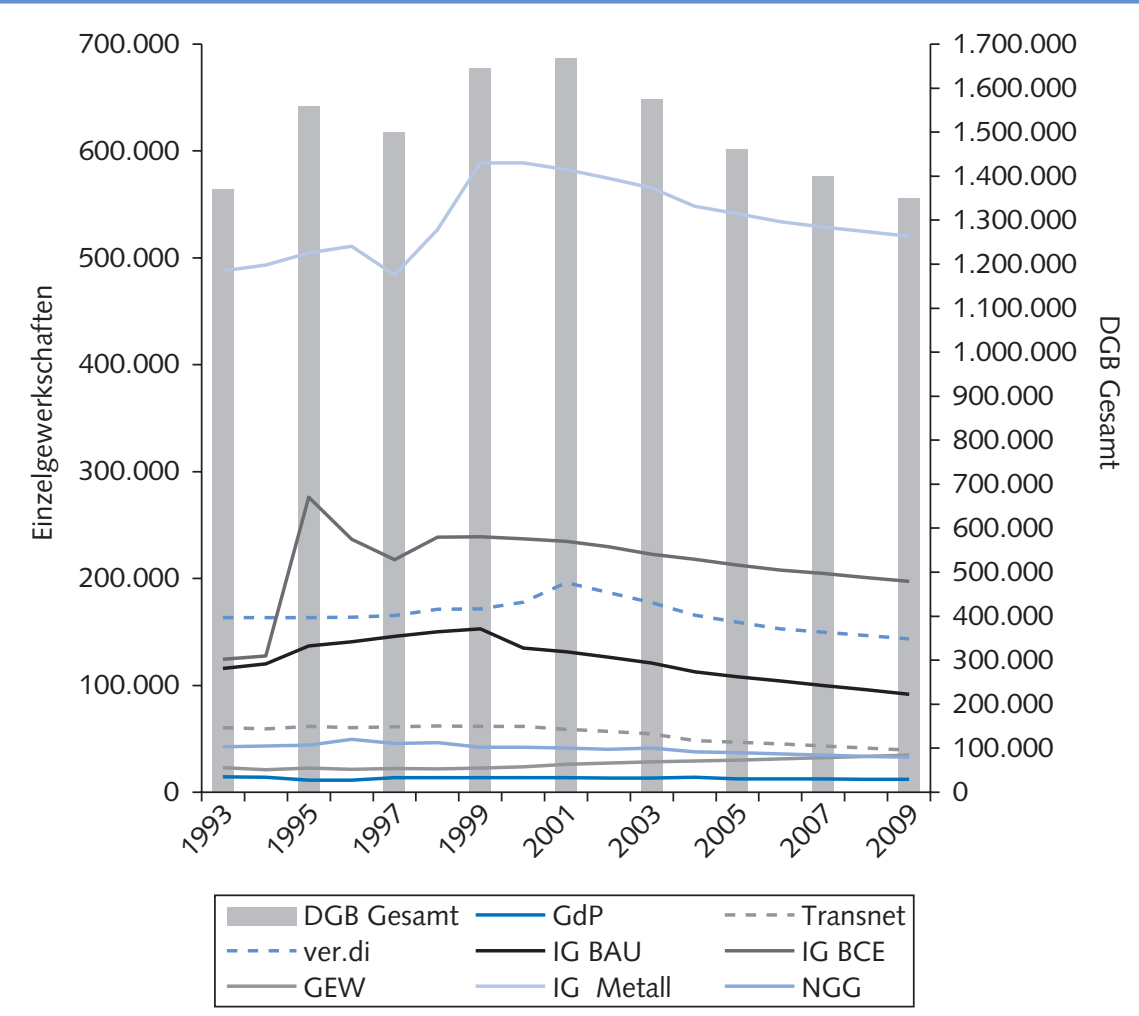

*Die Daten der Gründungsgewerkschaften sind vor den Fusionen zum Zweck einer besseren Darstellung summiert worden.

Quelle: DGB-Bundesvorstand; Darstellung der Autoren. die Zahl der Rentner im Vergleich zum Jahr 1995 abgenommen hat. Dagegen lag der Höhepunkt bei der IG Metall im Jahr 1999, der sich vor allem mit der Vorruhestandspolitik in der Metall- und Elektrobranche Ende der 1990er Jahre erklärt. Mit dem Zusammenschluss ihrer fünf Quellgewerkschaften verzeichnete ver.di im Jahr seiner Gründung (2001) den absoluten Höchststand an Seniorenmitgliedern (Abbildung 1).

Gründe dafür, dass die Entwicklung in den einzelnen Gewerkschaften unterschiedlich verläuft, liegen in den jeweils prägenden Unternehmens- und Berufskulturen, Traditionen sowie strukturellen Veränderungen des Branchensegments. Zugleich spielen aber auch die Angebote der einzelnen Gewerkschaften eine Rolle: TRANSNET war mit einem Rentner- bzw. Pensionärs-Anteilvon 43,8 \% im Jahr 2009 die „älteste“ Gewerkschaft im Deutschen Gewerkschaftsbund (DGB). ${ }^{7}$ Im Zuge der Umstrukturierung des Bahnsektors erfolgten nur wenige Neueinstellungen, somit traten auch nur wenige Mitglieder in die Gewerkschaft ein. Ein weiterer Grund für die verhältnismäßig alte Mitgliederstruktur ist in der traditionell tiefen Verbundenheit vieler Mitglieder zur ehemaligen Unternehmensgewerkschaft der Deutschen Bahn zu finden. Die fest verankerte Gewerkschaftsbindung bleibt auch im Ruhestand bestehen, somit verzeichnet TRANSNET lediglich eine „minimale Fluktuation“ (Müller/Wilke 2006; Wolf et al. 1994, S. 32). Während bei TRANSNET traditionell eine den Senioren freundlich gesonnene Organisationskultur existiert, kann eine ähnliche Anreizkultur im Baubereich nicht unterstellt werden: Trotzdem erhöhte sich der Seniorenanteil der IG BAU (Bauen, Agrar, Umwelt) seit 1995 um 9,2\% auf 28,1 \% im Jahr 2009 (vgl. hierzu und im Folgenden Tabelle 1). Damit zählen TRANSNET und IG BAU zu jenen Gewerkschaften, die im Zeitraum von 1995 bis 2009 am stärksten gealtert sind. Gemessen an der Mitgliedschaftsstruktur ist die Gewerkschaft Erziehung und Wissenschaft (GEW) die jüngste Organisation. Der Anteil von Mitgliedern im Ruhestand betrug dort

6 Das Altersteilzeitgesetz wurde 1996 eingeführt.

7 Die überalterte Mitgliederstruktur ist wohl auch ein Grund für die Fusion von TRANSNET und GDBA zur neuen Eisenbahn- und Verkehrsgewerkschaft (EVG) im Oktober 2010. 
2009 lediglich 13,5 \%. ${ }^{8}$ Die Gewerkschaften des öffentlichen Dienstes ver.di (Vereinte Dienstleistungsgewerkschaft), GdP (Gewerkschaft der Polizei) und GEW, die eine hohe Anzahl an Angestellten organisieren, weisen einen vergleichsweise geringen Seniorenanteil auf. Dies lässt sich darauf zurückführen, dass Angestellte im Allgemeinen weniger geneigt sind, sich gewerkschaftlich zu organisieren (Ebbinghaus 2003, S. 175) und folglich auch mit dem Austritt aus dem Berufsleben meist ihre Mitgliedschaft beenden. Der Anteil der pensionierten Beamten in den Mitgliedsgewerkschaften des DGB lag im Jahr 2009 bei rund 2,2 \%.

Der Blick auf die Industriegewerkschaften IG Metall und IG BCE zeigt einen verhältnismäßig moderaten Anstieg des Rentneranteils: Die IG Metall hatte 1995 einen Anteil von 19,6\%, der um 3,4\% auf $23 \%$ im Jahr 2009 anstieg. Vor ihrem Zusammenschluss zur IG BCE hatten die drei Quellgewerkschaften IG CPK, IG BE und GL zusammen einen Rentneranteil von $24,6 \%$, wobei dieser in der IG Bergbau mit 39,3 \% am höchsten war. Im Jahr 2009 wuchs der relative Anteil - trotz absoluter Verluste in dieser Gruppe - auf 28,7 \%. Der Anstieg des Seniorenanteils lässt sich neben den allgemeinen Mitgliederverlusten in der Erwerbstätigengruppe vor allem auch auf die in den 1980er und 90er Jahren betriebene Frühverrentungspraxis in den jeweiligen Branchen zurückführen.

Die Vertretungsmacht der Gewerkschaften basiert in erster Linie auf einem möglichst hohen Organisationsgrad, denn nur als mitgliederstarke Verbände können sie ihr primäres Organisationsziel, die Interessenvertretung der Erwerbstätigen, wirkungsvoll verfolgen (Streeck 1979; Ebbinghaus 2003, S. 175). Mit Blick auf ihr numerisches Gewicht in der politischen Organisationslandschaft haben sie aber auch ein grundsätzliches Interesse daran, dass Mitglieder nach ihrem Übergang in den Ruhestand an die Organisation gebunden bleiben. Es ist also weniger die Zahl der Senioren, die die Gewerkschaften herausfordert, vielmehr sind es die gestiegenen Partizipationserwartungen der Älteren an angemessene Organisations- und Integrationsangebote. Wie bereits Kohli, Künemund und Wolf festhielten, steht neben den tradierten Formen der stellvertretenden Interessenvertretung für die ehemals Beschäftigten als „Politik für Ältere“ und

Tabelle 1: Gewerkschaftssenioren** in den DGB-Gewerkschaften 1995 und 2009 im Vergleich

\begin{tabular}{|c|c|c|c|c|c|c|}
\hline Gewerkschaft & $\begin{array}{c}1995^{*} \\
\text { Mitglieder } \\
\text { Insgesamt }\end{array}$ & $\begin{array}{l}\text { Senioren } \\
\text { mitglieder }\end{array}$ & $\begin{array}{l}\text { Senioren } \\
\text { in } \%\end{array}$ & $\begin{array}{c}2009^{*} \\
\text { Mitglieder } \\
\text { insgesamt }\end{array}$ & $\begin{array}{l}\text { Senioren } \\
\text { mitglieder }\end{array}$ & $\begin{array}{l}\text { Senioren } \\
\text { in \% }\end{array}$ \\
\hline IG BAU & 722.576 & 136.874 & 18,9 & 325.421 & 91.524 & 28,1 \\
\hline IG BCE & 1.122 .687 & 276.178 & 24,6 & 687.111 & 197.254 & 28,7 \\
\hline GEW & 306.448 & 22.676 & 7,4 & 285.119 & 34.758 & 13,5 \\
\hline IG Metall & 2.869 .469 & 526.799 & 19,6 & 2.263 .020 & 520.252 & 23 \\
\hline NGG & 322.019 & 44.150 & 13,7 & 204.670 & 32.655 & 16 \\
\hline GdP & 198.897 & 27.075 & 13,6 & 169.140 & 29.641 & 17,5 \\
\hline TRANSNET & 398.404 & 149.250 & 37,5 & 219.242 & 96.020 & 43,8 \\
\hline ver.di & 3.026 .974 & 397.288 & 13,1 & 2.138 .200 & 348.586 & 16,3 \\
\hline DGB-Gesamt & 9.354 .670 & 1.558 .235 & 16,7 & 6.264 .923 & 1.350 .690 & 21,6 \\
\hline \multicolumn{7}{|c|}{$\begin{array}{l}\text { * jeweils zum Jahresende. } \\
\text { *** Als Gewerkschaftssenioren werden diejenigen Gewerkschaftsmitglieder bezeichnet, die aus Altersgründen aus dem aktiven } \\
\text { Erwerbsleben ausgeschieden und Bezieher von gesetzlichen Rentenleistungen sind. }\end{array}$} \\
\hline \multicolumn{7}{|c|}{ Quelle: DGB-Bundesvorstand; Berechnungen der Autoren. } \\
\hline
\end{tabular}

die (nicht unbedingt seniorenspezifische) Mitarbeit Älterer in der Organisation als „Politik von Älteren“ zunehmend der Anspruch einer Selbstorganisation der Älteren im Raum. In diesem Sinne streben Teile der ,jungen Alten“, insbesondere die 55bis 69-Jährigen, eine Interessenvertretung als „Politik von Älteren für Ältere“ (Künemund et. al 1993; Wolf et. al 1994; Kohli et al. 1997) innerhalb der Gewerkschaften an.

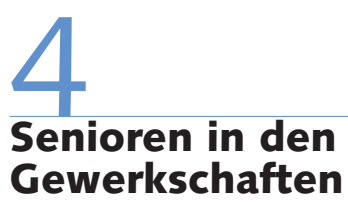

\subsection{BEGRENZTE INTEGRATION IN DER GESAMTSCHAU}

Die Gewerkschaften verstehen sich grundsätzlich als generationenübergreifende "Institutionen der Solidarität“ (Schroeder/Keudel 2008, S. 15), die „auf dem Grundsatz der gegenseitigen Hilfe“ beruhen (Neumann 1978, S. 150). Dieses Selbstverständnis schließt auch die Interessenvertretung der ehemals Beschäftigten mit ein. So sind die Gewerkschaften als Tarifpartei durch die strukturelle Kopplung der Renten an die Bruttolöhne ${ }^{9}$ unmittelbar an der Entwicklung der Altersbezüge beteiligt. Ebenso nehmen sie durch ihre Vertretungsfunktion in den Selbstverwaltungsgremien der Sozialversicherungen Einfluss auf die Alterssicherung und die Gesundheitsversorgung der Älteren. Doch diese Politik für Ältere findet vornehmlich ohne die direkte Beteiligung der Älteren selbst statt.

Die Mitglieder im Ruhestand sind in den meisten Gewerkschaften formal kei- ne Vollmitglieder, denn sie zahlen einen verminderten Mitgliedschaftsbeitrag und sind in den gewerkschaftlichen Entscheidungsgremien kaum direkt vertreten. Dem stehen tendenziell gestiegene Partizipationsansprüche einer größer gewordenen Zahl aktiver Gewerkschaftssenioren gegenüber (vgl. auch Bundesarbeitskreis Arbeit und Leben 2002), die mehr Beteiligungsrechte (Satzungsbestimmungen, Personengruppenstatus, Antragsrechte, Richtlinien etc.) einfordern. Vorrangig lassen sich diese Aktivitäten vor allem als eine Möglichkeit dieser Mitgliedergruppe interpretieren, Kontinuität der individuellen Lebensbezüge herzustellen und eine Anerkennung für ihre langjährige Gewerkschaftszugehörigkeit zu erhalten. Zugleich spielt auch die Konkurrenz zu den Angeboten der Sozialverbände, der Kommunen, vereinzelt auch der Parteien eine Rolle. Die Gewerkschaften tragen diesem Anliegen in Gestalt einer Beteiligung in seniorenspezifischen Gremien bislang nur begrenzt und in unterschiedlicher Form Rechnung: In sechs der acht DGB-Gewerkschaften können Rentner bzw. Pensionäre sich als

8 Dieser vergleichsweise niedrige Anteil ist auf die Struktur der Alterskohorten sowie auf die Einstellungspolitik der Kultusministerien der Länder zurückzuführen. Im Jahr 2007 stellten die Mitglieder im Alter zwischen 46 und 55 Jahren rund ein Drittel, die Gruppe der 56- bis 65-Jährigen $26,9 \%$ der Gesamtmitgliedschaft. Letzte genannte Gruppe ist im Zuge der Einstellungswelle in den 1970er Jahren in die GEW eingetreten. In den kommenden Jahren wird diese Gruppe aus dem Erwerbsleben ausscheiden, sodass der Rentnerund Pensionärsanteil der GEW zunehmen wird.

9 Dieses Koppelungsprinzip gilt nach wie vor be steigenden Löhnen. Das 2009 verabschiedete Rentensicherungsgesetz verhindert allerdings, dass die Renteneinkommen bei sinkenden Bruttolöhnen gleichfalls abgesenkt werden. 
eigene Mitgliedergruppe in Gremien und Ausschüssen auf allen Organisationsebenen zusammenschließen. Die Industriegewerkschaften IG BCE und IG Metall halten sich bei einer formell stärkeren Einbindung der Gewerkschaftssenioren zurück.

Auffällig ist, dass vor allem die Gewerkschaften im öffentlichen bzw. des ehemaligen öffentlichen Dienstes verhältnismäßig breite Vertretungsstrukturen für Gewerkschaftssenioren aufweisen. Auf diese Weise scheint es ihnen zu gelingen, ältere Mitglieder auch in der Ruhestandsphase an sich zu binden. Beamte, deren Organisationsgrad traditionell hoch ist (Ebbinghaus 2003, S. 190), sind eher bereit, auch als Pensionäre ihre Mitgliedschaft aufrechtzuerhalten, wie die Erfahrungen bei den ehemaligen Staatsunternehmen der Deutschen Bahn und Post belegen. ${ }^{10}$ Dagegen erscheint es schwierig, Angestellte in der Privatwirtschaft, vor allem im Dienstleistungssektor, langfristig an die Gewerkschaft zu binden. So ist zumindest der Eindruck, wenn man ver.di als Vergleich heranzieht, deren Seniorenanteil - trotz breiter Formalrechte für diese Mitgliedergruppe - rund $16 \%$ beträgt. ${ }^{11}$ Gewerkschaftliche Seniorenarbeit ist eher dort vorfindbar, wo entweder Beamte organisiert sind oder aber traditionsreiche Industrieberufe vorherrschen und sich parallel dazu ein langjähriges Zugehörigkeitsgefühl zu großen Betrieben bei den Beschäftigten herausbilden konnte. Dort, wo berufsethische Motive nur rudimentär bestehen und ein Berufs- oder Betriebsstolz fehlt, ist auch das Senioritätsprinzip als Teil der Organisationskultur und damit als Voraussetzung für eine ausgeprägte gewerkschaftliche Seniorenarbeit kaum vorhanden. Im Folgenden wird ein Überblick über die Einbindung der Gewerkschaftssenioren im DGB und in den Einzelgewerkschaften IG Metall, ver.di und IG BCE gegeben.

\subsection{EINZELBETRACHTUNGEN: GEWERKSCHAFTSSENIOREN IN IG METALL, VER.DI, IG BCE UND DGB}

Jede Gewerkschaft hat eigene Vorstellungen darüber, wie ihre Mitglieder im Ruhestand in die Organisation eingebunden werden. Diese Vielfalt ist vor dem Hintergrund der jeweiligen historisch gewachsenen Organisationskulturen zu verstehen. So verfügen etwa in ver.di die Senioren als satzungsverankerte Personengruppe über weitreichende Beteiligungsstrukturen.
Die Aktivitäten der in Rente befindlichen IG Metall-Mitglieder sind hingegen nicht in der Satzung formal festgeschrieben. In der IG Metall ist die Seniorenarbeit ein Bestandteil der „Außerbetrieblichen Gewerkschaftsarbeit" (AGA). Diese Formalisierung ist als ein Ergebnis des IG Metall internen Organisatons-Enwicklungs-Prozesses (OE) zu sehen. Dieser Prozess, der in den 1990er Jahren beginnend auf den Abbau von Sondergruppeninteressen setzte, hatte zur Folge, dass Rentner, Erwerbslose, Studierende, Schüler und Mitglieder ohne betriebliche Betreuung fortan in der AGA zusammengefasst werden. Dagegen sind die Gewerkschaftssenioren in ver.di eine satzungsverankerte Personengruppe. Diese formale Einbindung ist ein Ergebnis des Fusionsprozesses. Das Beibehalten der Strukturen aus den Gründungsgewerkschaften, insbesondere der ehemaligen Unternehmensgewerkschaft DPG (Deutsche Postgewerkschaft), war eine Folge der Kompromissfindung. Die Seniorenarbeit in ver.di ist je nach Fachbereich unterschiedlich stark ausgeprägt und steht in der Kontinuität der jeweiligen Quellgewerkschaft. Rentner stellen in der IG BCE mittlerweile rund ein Viertel der Gesamtmitgliedschaft. Die Bindung vieler Mitglieder im Ruhestand beruht auf der Bedeutung der betrieblichen Sozialpolitik in der Großchemie und im Bergbau. Satzungsformell besitzen sie innerhalb der Organisation hingegen keinen gesonderten Status. Aus der Tradition der ehemaligen IG BE behalten Mitglieder auch nach Beendigung ihrer Erwerbstätigkeit ihre vollen Rechte. In den wohnortsnahen Ortsgruppen engagieren sich vorwiegend Rentner, ohne dass deren Aktivitäten explizit als Seniorenarbeit bezeichnet werden. In den Bereichen der ehemaligen IG CPK bestehen Seniorenarbeitskreise meist angesiedelt an den chemischen Großunternehmen. Die formelle Gleichbehandlung mit den beschäftigten Mitgliedern und die Möglichkeit zu örtlichen Seniorenaktivitäten wirkt der Forderung nach mehr Mitspracherechten vonseiten der Rentner entgegen.

Rentner und Pensionäre können sich sowohl in eigenen Gremien des Dachverbandes (DGB) wie auch der Einzelgewerkschaften engagieren. Zugleich gibt es Friktionen und Unklarheiten in der Aufgabenteilung der Seniorenpolitik. Nach Definition des Dachverbandes sollte Seniorenarbeit in der jeweiligen Einzelgewerkschaft stattfinden, während Seniorenpolitik das primäre Koordinationsfeld des DGB sein sollte. Eine direkte, jedoch kontrollierte Interessenvertretung durch die Senioren erscheint vor allem deshalb erforderlich, um einerseits den Forderungen der Gewerkschaftssenioren nachzukommen, andererseits um in den sozialpolitischen Reformdebatten als generationenübergreifender Akteur auftreten zu können. Der DGB tut sich jedoch schwer, ein klares Strategiekonzept für diese explizite Interessenvertretung durch die Senioren und eine entsprechende Einbindung zu entwickeln. Auch wird die Unterscheidung zwischen Seniorenarbeit und Seniorenpolitik nicht konsequent verfolgt. Die Hintergründe dieser Unübersichtlichkeit, die sich in einer muddling-through-Strategie äußert, sind darin zu sehen, dass die verantwortlichen Akteure mit einer intensiven, auch öffentlich geführten Auseinandersetzung über die explizite Interessenvertretung der Gewerkschaftssenioren einen möglichen Imageverlust als starke Arbeitnehmerorganisation fürchten (vgl. Schroeder et al. 2010, S. 319ff.). Ebenso sollen daraus sich ergebende Konsequenzen für die Organisationsstruktur des DGB und damit einhergehende Kosten vermieden werden.

Um nicht an Profilschärfe als Interessenvertretung der beschäftigten Arbeitnehmer zu verlieren und dennoch advokatorisch im Feld der Seniorenpolitik aktiv zu sein, ging der DGB in den vergangenen Jahren wiederholt Bündnisse mit den Sozialverbänden ein (vgl. ebd.). Die Zusammenarbeit basiert überwiegend auf konkreten sozialpolitischen Sachfragen. Auf diese Weise wird ein wechselseitiger Nutzen der Kooperationspartner angestrebt, ohne dafür eigene Strukturen aufzubauen und zusätzliche Ressourcen zu verbrauchen. Organisationspolitisch könnte eine institutionalisierte Zusammenarbeit mit den Sozialverbänden auch weitere Vorteile bringen, indem beispielsweise eine geteilte Mitgliedschaft für Senioren dazu beitragen könnte, deren Interessen aufzuteilen und sie so besser abzudecken.

Die Gesamtentwicklung der vergangenen Jahre zeichnet sich zum einen

10 Der im DBB organisierte Seniorenverband BRH (Bund der Ruhestandsbeamten, Rentner und Hinterbliebenen) organisiert rund 80.000 Pensionäre und Rentner, die Mitglieder des DBB sind bzw. waren.

11 In ver.di sind rund ein Drittel Arbeiter, die Hälfte Angestellte und rund $10 \%$ Beamte organisiert. 
durch einen sukzessiven Abbau materieller Satzungsleistungen aus. Zum anderen sind mehr oder weniger ausgeprägte Beteiligungsmöglichkeiten für Senioren in den meisten Gewerkschaften zumindest formell etabliert worden. Letzteres kann als Zeichen dafür gewertet werden, dass man bestrebt ist, das Selbstverständnis und das politische Handeln als generationenübergreifende Organisation unter dem demografischen Veränderungsdruck aufrechtzuerhalten. Zumindest soweit wie die Interessensvertretung der erwerbstätigen Mitglieder nicht beschnitten wird. Hier lässt sich beobachten, dass interessenpolitische Zugeständnisse an die Senioren meist umso größer ausfallen, je stärker diese Mitgliedergruppe ein klientelbezogenes Interessenbewusstsein besitzt und dieses artikuliert wird.

\section{Die Seniorenfrage als innergewerkschaftlicher Zielkonflikt?}

Wenn sich das numerische Verhältnis zwischen beschäftigten und nicht mehr erwerbstätigen Mitgliedern zugunsten Letzterer verschiebt, so könnte dies auch Folgen für die (nach außen gerichtete) gewerkschaftliche Strategie- und Zieldefinition im Sinne einer einheitlichen Mitglieder- und Organisationslogik ergeben. So zumindest bewerten handelnde Akteure in den Gewerkschaften die Seniorenfrage. Diese verstehen wir als organisationale Herausforderung in der Weise, dass die Gewerkschaften als erwerbszentrierte, intermediär agierende Organisation Handlungsperspektiven entwickeln müssen, um mit einer alternden Mitgliederstruktur im Rahmen ihrer politischen Zielfunktion umzugehen. Doch der entscheidende Punkt ist weniger die Quantität, sondern vielmehr die Qualität. Oder anders formuliert: So lange ein hoher Anteil nicht erwerbstätiger Mitglieder weder Beteiligungsansprüche geltend macht noch als geschlossene Gruppe seniorenspezifische Interessen artikuliert, ist diese Entwicklung für die Gewerkschaften unproblematisch. Zum Problem kann die Seniorenfrage dann werden, wenn sich die Senioren als machtpolitischer Block mit eigenen Positionen in den innerorganisationalen Meinungs- und Entscheidungsprozess derart einbringen, dass die gewerkschaftlichen Grundanliegen gefährdet werden.

Die Ausrichtung und Orientierung am Primat der Erwerbsarbeit ist bislang eng mit dem sozialpolitischen Modell einer generationsübergreifenden Sozialpolitik als „Politik für alle Lebensalter“ verkoppelt. So ließen sich die sozialpolitischen Interessen beider Gruppen zu Zeiten der Expansionsphase des Wohlfahrtsstaates im Sinne des solidarischen Generationenvertrages ausbalancieren (Schölkopf 2000). Mit der wegweisenden Rentenreform im Jahr 1957, an der die Gewerkschaften als politische Akteure maßgeblich mitgewirkt haben, wurde ihr generationenübergreifendes Selbstverständnis auch von staatlicher Seite anerkannt und so die advokatorische Interessenvertretung der Älteren durch die Gewerkschaften institutionalisiert: Im Zuge der Einführung der bruttolohnbezogenen, dynamischen und lebensstandardsichernden Rente nach dem Umlageverfahren wurden die jährlichen Anpassungen der Rentenhöhe an die Lohnentwicklung geknüpft, die unabhängig von staatlicher Kontrolle zwischen den Tarifpartnern ausgehandelt wird. Damit konnten sich die Gewerkschaften im Rahmen ihrer Tarifverhandlungen auch als advokatorische Interessenvertreter der materiellen Interessen ihrer Randklientel, der verrenteten bzw. pensionierten Mitglieder, legitimieren und eine exklusive Stellung im Verbändesystem erlangen. Für ihre verrenteten Mitglieder handeln sie folglich im Sinne einer advokatorischen Kraft (vgl. ebd., S. 125; Willems/Winter 2000, S. 25f.), ohne dass sie einen expliziten Einfluss auf die inhaltliche Ausgestaltung der generationenübergreifenden Politik nehmen können.

Gleichwohl sind mit dem Um- und Rückbau des Sozialstaats der vergangenen Jahre neue Konfliktlinien entstanden. Die Dezimierung sozialer Leistungen hat zu divergierenden Interessenlagen in der Sozialstaatsdebatte geführt. Während aktiv Beschäftigte die Begrenzung der Abgabenlast durch stabile Beitragssätze und die Sicherung der eigenen Zukunftsinteressen anstreben, haben die gegenwärtigen Rentenbezieher ein Interesse an der Stabilität des bestehenden Rentenniveaus - unter Umständen auch durch Beitragserhöhungen, die zulasten der Erwerbstätigen gehen würden. Es hat den Anschein, als ob die Gewerkschaften auch deshalb eine defensive Haltung zur Verteidigung des Status quo einnehmen, um möglichen Konflikten in den eigenen Reihen entgegenzuwirken. Grundsätzlich besteht also die Gefahr, dass das Spannungsverhältnis von Mitgliederund Einflusslogik (Schmitter/Streeck 1981) ein zunehmend schwieriger werdendes Vermittlungsverständnis zwischen „interner und externer Interessenwahrnehmung" (Esser 2003, S. 68) zur Folge hat.

Die Kosten für ein erweitertes Vertretungsmandat werden von den Führungsakteuren der Einzelgewerkschaften wie auch des DGB als hoch eingeschätzt. Neben den dafür erforderlichen finanziellen Ressourcen werden auch Steuerungs- und Abstimmungsprobleme für die interne Ausbalancierung der Interessenlagen antizipiert. Vor dem Hintergrund der begrenzten finanziellen Ressourcen seien die Gewerkschaften gefordert, sich auf ihre Kernaufgaben, namentlich die Vertretung der aktiv beschäftigten „Stammkunden“ (Streeck 1987) in den arbeits- und tarifpolitischen Arenen zu konzentrieren. Aber vor allem scheint es aus der Funktionärsperspektive einen strategischen Zielkonflikt zu geben: Nämlich zwischen dem Interesse, den Arbeitsmarkt breit gewerkschaftlich zu organisieren, um so attraktiv für jüngere Arbeitnehmer zu sein, und sich gleichzeitig für die Älteren zu öffnen. Befürchtet wird, mit einer zu aktiven Rolle der Älteren das Profil als starker, vitaler politischer Kampfverband zu schwächen. Diese Bedenken gründen unter anderem auf dem nach wie vor weit verbreiteten defizitorientierten Altersbild. Die formale Einbindung und die damit einhergehende Institutionalisierung von expliziten Vertretungs- und Mitspracherechten in der Organisation birgt somit durchaus die Gefahr, dass aus latenten manifeste Funktionswidersprüche werden. Die strategischen Akteure in den Gewerkschaften sind bestrebt, die mit der organisationsinternen Pluralisierung und Heterogenisierung der Interessen neu entstehenden Konfliktlinien innerhalb des vorhandenen, auf Homogenität zielenden institutionellen Arrangements zu bearbeiten.

Die Seniorenfrage steht nicht zentral auf der Agenda der deutschen Gewerkschaften; sie scheint auch zum gegenwärtigen Zeitpunkt kein manifestes, also drängendes Konfliktpotenzial zu beinhalten. Die Gewerkschaften verhalten sich in dieser Frage bislang eher behutsam, zurückhaltend. Gleichwohl ist ihnen die Frage nicht gleichgültig, wie viele Senio- 
ren organisiert sind, welche Aufgaben sie wahrnehmen und welche Rolle sie spielen. Denn ohne diese Mitgliedergruppe würde bspw. neben dem Wegfall der Beitragseinnahmen auch ein, in vielen Fällen aber auch noch ungenutztes, Potenzial an ehrenamtlicher Unterstützung fehlen. Das Engagement der Senioren findet auf örtlicher Ebene statt - dort leisten Senioren mancherorts einen Beitrag bei der Bewältigung der alltäglichen, arbeitsorganisatorischen Aufgaben und können auf diese Weise hauptamtliche Mitarbeiter entlasten. Nicht zu unterschätzen ist auch ihr aktiver Beitrag für die Legitimation als generationenübergreifende Organisation. Schließlich speist sich die gewerkschaftliche Interessenvertretungslogik nicht nur durch die Solidarität zwischen den Erwerbstätigen in unterschiedlichen Unternehmen, Branchen und Regionen, sondern eben auch durch die Solidarität zwischen den Generationen.

Der Nutzen einer stärkeren, konditionierten Einbindung der Gewerkschaftssenioren ist auch vor dem Hintergrund ihres sozialen Kapitals - in Form ihres meist langjährigen Erfahrungsschatzes und ihrer Kompetenzen - zu sehen. Als „stille Reserve" beteiligen sie sich häufig an Demonstrationen und Warnstreiks, sind mit Handlungsabläufen vertraut und können hauptamtlich Beschäftigte unterstützen. Als Multiplikatoren mit Zeitressourcen, die sowohl in soziale Netzwerke vor Ort eingebettet als auch in den Organisationen rückgebunden sind, übernehmen sie durch ihr ehrenamtliches Engagement eine wichtige, intergenerative Vermittlerrolle. Möchte man weiterhin möglichst breit in der Fläche präsent sein, so könnte diese Gruppe, wenn sie sich unter definierten
Bedingungen einbringen kann, eine zusätzliche Chance sein, um Vertretungslücken zu kompensieren. Kurzum: Durch den Verbleib der Mitglieder im Ruhestand erhalten Gewerkschaften materielle und ideelle Unterstützung für ihre Arbeit und können einen höheren Legitimations- und Repräsentationsanspruch für sich reklamieren.

\section{6 \\ Schlussbetrachtung}

Eine ernsthafte Debatte über eigenständige Rentnergewerkschaften hat es in Deutschland nie gegeben. Und die $\mathrm{Zu}-$ kunftsfähigkeit der Gewerkschaften hängt nicht von der Mitgliedergruppe der Senioren $\mathrm{ab}$ - ja prinzipiell könnten sie sogar ohne diese außerbetriebliche Mitgliedergruppe auskommen. Um ihren Bestand $\mathrm{zu}$ sichern und zu revitalisieren, müssen Gewerkschaften strategisch vielmehr die Rekrutierung jüngerer, erwerbstätiger Zielgruppen verfolgen. Zudem haben die Gewerkschaften in den letzten Jahren wenig getan, um Rentnern und Pensionären ein attraktives Angebot für den Organisationsverbleib sowie für ein aktives Engagement in den Gewerkschaften $\mathrm{zu}$ unterbreiten. Im Gegenteil: Unterstützungsleistungen für Senioren sind in den letzten 16 Jahren deutlich abgebaut worden.

Hinsichtlich der Unterschiede, die zwischen den Gewerkschaften bestehen, ist es auffallend, dass insbesondere in den unternehmensnahen Gewerkschaften nicht nur das Senioritätsprinzip im Arbeitsleben, sondern auch der Verbleib und das
Engagement im Ruhestand eine andere Selbstverständlichkeit besitzen.

Es gibt nicht nur Befürchtungen, dass ein zu starkes Engagement der Senioren belastend für die Attraktivität des dynamischen Erscheinungsbildes der Gewerkschaft wirken kann. Es gibt vor allem vielfältige konkrete Erfahrungen, dass Rentner in den Organisationen als Kräfte der Beharrung wirken können, die notwendige Veränderungsprozesse eher behindern. Doch trotz geäußerter Zielkonflikte und zuweilen schwieriger Debattenlagen gehen die Gewerkschaften mit der Seniorenfrage sensibel um. Denn ihre funktionale, strategische Ausrichtung am Primat der Erwerbsarbeit erfordert eine bedachtsame Interessenausbalancierung zwischen Erwerbstätigen und Mitgliedern im Ruhestand. Ein Weg, der in dieser Frage gegangen wurde, ist die Formalisierung der ehrenamtlichen Seniorenarbeit. In allen untersuchten Gewerkschaften sind Mitglieder im Ruhestand seit Jahrzehnten vor Ort aktiv, ihr Engagement besaß in manchen Gewerkschaften lange informellen Charakter. Doch durch die Formalisierung der Seniorenarbeit in eigenen Gremien eröffnen sich für die aktiven Älteren Beteiligungsräume, die für beide Seiten - also für die erwerbstätigen und die nichterwerbstätigen Mitglieder - einen Nutzen stiften können. Zukünftig stellt sich für die Gewerkschaften die Frage, wie sie dieses Engagementpotenzial der aktiven Älteren systematischer nutzen können. Flankiert werden müsste dies allerdings mit einer Organisationskultur, die dem Wunsch nach Anerkennung der individuellen Lebensleistung und biografischer Kontinuität auch im Ruhestand nachkommt. 
Bundesarbeitskreis Arbeit und Leben (2002): Bildung für ein gemeinwesenorientiertes freiwilliges Engagement im Alter. Bedarfserhebung: Qualifizierung von älteren gewerkschaftlich orientierten Personen zur ehrenamtlichen Tätigkeit, Düsseldorf

Ebbinghaus, B. (2003): Die Mitgliederentwicklung deutscher Gewerkschaften im historischen Vergleich, in: Schroeder, W./Wessels, B. (Hrsg.): Die Gewerkschaften in Politik und Gesellschaft der Bundesrepublik Deutschland, Wiesbaden, S. 174-203

Esser, J. (2003): Funktion und Funktionswandel der Gewerkschaften in Deutschland, in: Schroeder, W./Wessels, B. (Hrsg.): Die Gewerkschaften in Politik und Gesellschaft der Bundesrepublik Deutschland, Wiesbaden, S. 65-85

Kohli, M./Künemund, H. (1998): Die Gewerkschaften im Konflikt um den Generationenvertrag, in: Gewerkschaftliche Monatshefte 49 (11)

S. $728-734$

Kohli, M./Künemund, H./Wolf, J. (1997): Trade unions and the elderly population: Is retirement still link to the sphere of work?, in: Scandinavian Journal of Social Welfare (6), S. 180-188

Künemund, H./Neckel, S./Wolf, J. (1993): Auf dem Weg zur Rentnergewerkschaft? Die Älteren als Herausforderung für gewerkschaftliche Politik, in: Leif, Th./Klein, A./Legrand, H.-J. (Hrsg.): Reform des DGB. Herausforderungen, Aufbruchpläne und Modernisierungskonzepte, Köln, S. 332-360

Müller, H.-P./ Wilke, M. (2003): Gestaltend Einfluss nehmen. Bahngewerkschaft und Bahnreform 1993-2005, Berlin

Neumann, F. L. (1978): Die Gewerkschaften in der Demokratie und in der Diktatur, in: Söllner, A. (Hrsg.): Wirtschaft, Staat, Demokratie. Aufsätze 1930-1954, Frankfurt am Main, S. 145-222

Pfahl, S./Reuyß, St. (2007): „GEW Mitgliederbindung im mittleren Lebensalter ", Ergebnisse der Studie, SowiTra, Berlin

Schmitter, P. C./Streeck, W. (1981): The organization of business interests: a research design to study the associative action of business in the advanced industrial societies of Western Europe, hrsg. vom Wissenschaftszentrum Berlin, Discussion Paper IIM/LMP (13), Berlin Schölkopf, M. (2000): Politisch repräsentiert oder ausgegrenzt? Pflegebedürftige ältere Menschen und die organisierten Interessen, in: Willems, U./Winter, Th. von (Hrsg.): Politische Repräsentation schwacher Interessen, Opladen, S. 113-148
Schroeder, W./Weßels, B. (Hrsg.) (2003): Die Gewerkschaften in Politik und Gesellschaft der Bundesrepublik Deutschland. Ein Handbuch, Wiesbaden

Schroeder, W./Keudel, D.(Hrsg.) (2008): Strategische Akteure in drei Welten. Die deutschen Gewerkschaften im Spiegel der neueren Forschung, Düsseldorf

Schroeder, W./Munimus/B./Rüdt, D. (2008): Integrierende oder separierende Interessenpolitik? Zum Selbstverständnis der Akteure der deutschen Seniorenpolitik - Sozialverbände, Gewerkschaften und Parteien im Vergleich, in: Zeitschrift für Sozialreform 54 (3),

S. $225-250$

Schroeder, W./Munimus, B./Rüdt, D. (2010): Seniorenpolitik im Wandel. Verbände und Gewerkschaften als Interessenvertreter der älteren Generation, Frankfurt am Main

Streeck, W. (1979): Gewerkschaften als Mitgliederverbände. Probleme gewerkschaftlicher Mitgliederrekrutierung, in: Bergmann, J. (Hrsg.): Beiträge zur Soziologie der Gewerkschaften, Frankfurt am Main, S. $72-110$

Streeck, W. (1987): Vielfalt und Interdependenz. Überlegungen zur Rolle von intermediären Organisationen in sich ändernden Umwelten, in: Kölner Zeitschrift für Soziologie und Sozialpsychologie 39 (4), S. 471-495

Streeck, W. (2007): Politik in einer alternden Gesellschaft: Vom Generationenvertrag zum Generationenkonflikt?, in: Gruss, P. (Hrsg.): Die Zukunft des Alterns. Die Antwort der Wissenschaft, München, S. 279-304 Willems, U./Winter, Th. von (2000): Die politische Repräsentation schwacher Interessen: Anmerkungen zum Stand und zu den Perspektiven der Forschung, in: Dies. (Hrsg.): Politische Repräsentation schwacher Interessen, Opladen, S. 9-38

Wolf, J./Bromberg, K./Ernst, F./Berndt, N./Kabel, M. (2003): Neue Ehrenamtlichkeit in traditionellen Mitgliederverbänden. Praxisfelder und Handlungspotentiale innovativer Formen des ehrenamtlichen Engagements, Forschungsbericht an die Hans-Böckler-Stiftung, http://www.jurgenwolf.de/docs/Titelblatt\%20und\%20Inhalt.pdf, (12.05.2007)

Wolf, J./Kohli, M./Künemund, H. (Hrsg.) (1994): Alter und gewerkschaftliche Politik. Auf dem Weg zur Rentnergewerkschaft?, Köln 